\title{
CLINICAL STUDY OF SIGMOID VOLVULUS
}

\author{
Vinod Babu $B^{1}$, Ajitha M. B2 \\ 1Postgraduate Student, Department of General Surgery, BMCRI. \\ ${ }^{2}$ Associate Professor, Department of General Surgery, BMCRI.
}

\begin{abstract}
\section{BACKGROUND}

Sigmoid Volvulus is a rare but most important cause of intestinal obstruction. Sigmoid volvulus generally affects adults and is more common in males, typically presenting with pain abdomen, abdomen distention of sudden onset and constipation. Aetiology of sigmoid volvulus is multifactorial and controversial. Delay in diagnosis leads to serious complications like bowel gangrene, perforation, peritonitis and sepsis.

This prospective study was done to analyse sigmoid volvulus; its clinical features, predisposing factors and mode of treatment. This study also emphasises on surgical management and prognosis of this condition.
\end{abstract}

\section{MATERIALS AND METHODS}

This is a prospective study which included all the 35 patients admitted and treated for sigmoid volvulus in Victoria Hospital, Bangalore from July 1, 2015 to June 30, 2016.

\section{RESULTS}

There were 35 patients admitted and treated for sigmoid volvulus during the study period. The mean age of occurrence was 49 years, with predominantly male patients. All our patients were active and physically fit prior to the onset of sigmoid volvulus. Most patients belonged to poor socioeconomic status, were involved in strenuous work for their livelihood and most consumed diet mainly rich in roughage and bulky in quantity. Patients presented with array of physical findings, with abdominal distension in almost all patients. Mortality rate in our study was $11.42 \%$. Those who survived the episode were able to return to their earlier vocation.

\section{CONCLUSION}

Sigmoid volvulus is a rare but an important cause of large bowel obstruction, a surgical emergency requiring prompt diagnosis and treatment by detorsion before patient develops complications such as gangrene or perforation. Primary resection and anastomosis is a permanent curative procedure for sigmoid volvulus.

\section{KEYWORDS}

Complications, Intestinal Obstruction, Mortality, Sigmoid Volvulus, Surgery.

HOW TO CITE THIS ARTICLE: Babu VB, Ajitha MB.Clinical study of sigmoid volvulus. J. Evolution Med. Dent. Sci. 2016;5(78):58245827, DOI: $10.14260 /$ jemds/2016/1314

\section{INTRODUCTION}

Sigmoid Volvulus defined as abnormal twisting of sigmoid colon around its mesentery, is an infrequent cause of colonic obstruction. ${ }^{1}$ Sigmoid Volvulus was first described in 1836 by von Rokitansky. ${ }^{2}$ It remains rare yet an important cause of intestinal obstruction. ${ }^{3}$ It is the second most common cause of strangulating obstruction of colon, first being carcinoma as a cause of colonic obstruction. ${ }^{4}$ Sigmoid Volvulus generally affects adults with highest incidence seen between $4^{\text {th }}$ to $8^{\text {th }}$ decade of life. ${ }^{5}$

It is more common in males and occurs in the ratio ranging from 2:1 to 10:1.6 It is believed that a lax abdomen and wider pelvis which provides space of spontaneous untwisting are said to be the reason for lesser rates of sigmoid volvulus in females. ${ }^{7}$

Financial or Other, Competing Interest: None.

Submission 24-08-2016, Peer Review 18-09-2016,

Acceptance 23-09-2016, Published 29-09-2016.

Corresponding Author:

Dr. Vinod Babu $B$,

No. 3, $7^{\text {th }}$ Main Road,

Vasanth Nagar,

Bangalore-560052.

E-mail: babuvinod11@gmail.com

DOI: $10.14260 /$ jemds $/ 2016 / 1314$

\section{The Aetiology of Sigmoid Volvulus is}

A. Congenital: Idiopathic, narrow attachment of mesentery of sigmoid colon, congenital bands, long mobile loop of sigmoid colon, Hirschsprung disease and pseudo megacolon.

B. Acquired and predisposing factors: Postoperative adhesions, loaded colon resulting from chronic constipation, dietary-large and heavy course diet, old age and drugs such as anticholinergics, ganglion blockers, antiParkinsonian drugs and tranquilisers also have been said to produce megacolon or megacolon syndrome. ${ }^{8}$

In many instances, the aetiology and level of obstruction can be identified by radiological investigation. When a dilated loop of bowel is seen, clinicians have to identify the level of obstruction, the loop of dilated bowel proximal to it and also the undilated bowel distal to it, an abdominal radiograph will suffice for diagnosing the level of bowel obstruction.

Although surgical exploration itself is an accepted mode for diagnosing acute abdomen, accurate preoperative diagnosis will reduce the morbidity and mortality. Delay in diagnosis and treatment of sigmoid volvulus can lead to sigmoid ischaemia, infarction, peritonitis, and septicaemia, resulting in mortality of up to $60 \% .{ }^{9}$ In most instances, decompression can be done nonoperatively with insertion of a rectal tube, or performing flexible sigmoidoscopy. ${ }^{10}$ However, 
sigmoidoscopy should not be performed in patients who have developed clinical evidence of bowel gangrene, perforation, peritonitis or sepsis.

Emergency laparotomy and resection with or without primary anastomosis is indicated when nonoperative methods fail, or when there is evidence of strangulation, infarction or perforation. ${ }^{11}$ Postoperative mortality ranges from $6 \%$ to $60 \%$.

\section{METHODOLOGY}

\section{Source of Data}

This prospective study included all the patients admitted and treated in all surgical units, Victoria Hospital, Bangalore between July 1, 2015 to June 30, 2016 for sigmoid volvulus.

The study included 35 cases of sigmoid volvulus, carried out as regards to the aetiological factors which predispose to sigmoid volvulus, the clinical features, mode of treatment and the outcomes. The duration of study was 1 year.

\section{Inclusion Criteria}

All patients who presented to Casualty at Victoria Hospital, BMCRI, Bangalore, with large bowel obstruction due to sigmoid volvulus.

\section{Exclusion Criteria}

All cases of large bowel obstruction other than sigmoid volvulus were excluded.

\section{RESULTS}

Male and female affected were 25 and 10 cases respectively. It would appear that in the study, sigmoid volvulus occurs almost with equal frequency between age groups 21-30 yrs., $31-40$ yrs. and 41-50 yrs. and these groups accounted for $48.4 \%$ of patients. The maximum number belonged to 51-60 age group i.e. $28.57 \%$ [Table 1].

All our patients were active and physically fit prior to onset of sigmoid volvulus. Majority of patients were involved in strenuous work for their livelihood [Table 2].

All patients belonged to poor socioeconomic status and most consumed diet mainly rich in roughage and bulky in quantity.

In present study, distension of abdomen [100\%] was most common symptom followed by constipation [94\%] and then pain abdomen [75\%] [Table 3].

When the bowel was viable, majority of the patients survived irrespective of surgical procedure carried out which accounted for $88.5 \% .4$ patients [11.42\%] died in the hospital [Table 4].

\begin{tabular}{|c|c|c|}
\hline Age [In years] & Total No. of Patients & Percentage \\
\hline $11-20$ & 2 & $5.7 \%$ \\
\hline $21-30$ & 5 & $14.2 \%$ \\
\hline $31-40$ & 5 & $14.2 \%$ \\
\hline $41-50$ & 7 & $20 \%$ \\
\hline $51-60$ & 10 & $28.57 \%$ \\
\hline $61-70$ & 5 & $14.2 \%$ \\
\hline $71-80$ & 1 & $2.8 \%$ \\
\hline \multicolumn{2}{|c|}{ Table 1: Age Distribution of Cases } \\
\hline
\end{tabular}

\begin{tabular}{|c|c|c|}
\hline Occupation & No. of Patients & Percentage \\
\hline Coolie & 20 & $57.14 \%$ \\
\hline Housewife & 10 & $28.57 \%$ \\
\hline Agriculture & 5 & $14.28 \%$ \\
\hline \multicolumn{2}{|r|}{ Table 2: Occupation of Patients } \\
\hline
\end{tabular}

\begin{tabular}{|c|c|c|}
\hline Symptoms & No. of Patients & Percentage \\
\hline Pain Abdomen & 26 & $74.28 \%$ \\
\hline Distension Abdomen & 35 & $100 \%$ \\
\hline Constipation & 32 & $91.42 \%$ \\
\hline Vomiting & 20 & $57.14 \%$ \\
\hline Retention of urine & 3 & $8.57 \%$ \\
\hline Fever & 1 & $2.85 \%$ \\
\hline \multicolumn{3}{|c|}{ Table 3: Mode of Presentation } \\
\hline
\end{tabular}

\begin{tabular}{|c|c|c|c|c|}
\hline 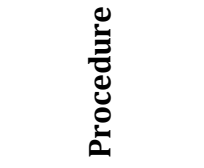 & 范 & $d^{0}$ & $\begin{array}{l}\bar{\Xi} \\
\stackrel{\Xi}{\Xi}\end{array}$ & 总 \\
\hline Sigmoidopexy & 12 & $34.28 \%$ & $\begin{array}{c}11 \\
{[91.6 \%]}\end{array}$ & $\begin{array}{c}1 \\
{[8.37 \%]}\end{array}$ \\
\hline $\begin{array}{l}\text { Primary } \\
\text { sigmoid } \\
\text { resection and } \\
\text { anastomosis }\end{array}$ & 15 & $42.85 \%$ & $\begin{array}{c}15 \\
{[100 \%]}\end{array}$ & $\mathrm{L}$ \\
\hline $\begin{array}{l}\text { Hartmann's } \\
\text { procedure }\end{array}$ & 8 & $22.85 \%$ & $\begin{array}{c}5 \\
{[62.5 \%]}\end{array}$ & $\begin{array}{c}3 \\
{[37.5 \%]}\end{array}$ \\
\hline
\end{tabular}

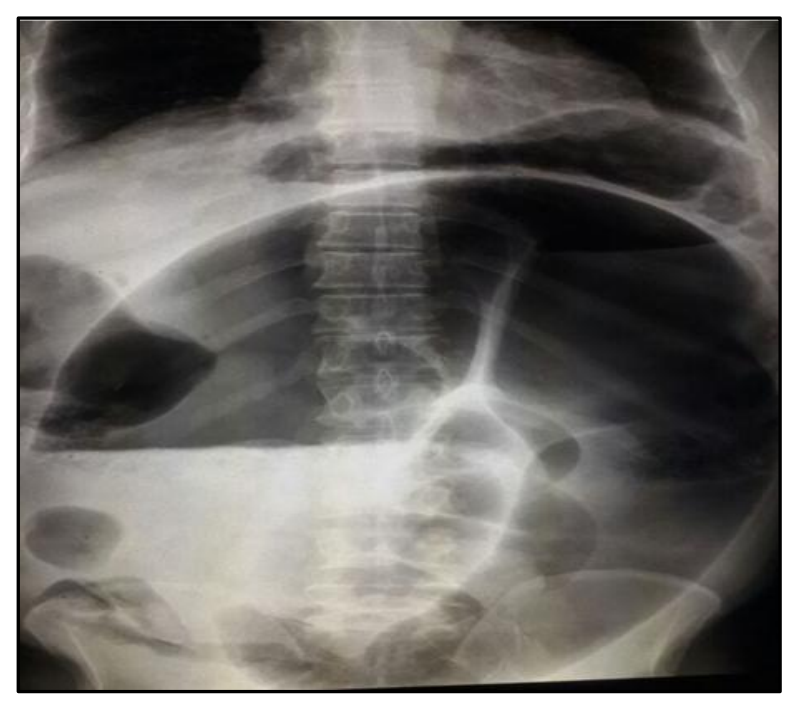

Image 1: Erect X-ray Abdomen showing Coffee Bean Sign (Sigmoid Volvulus)

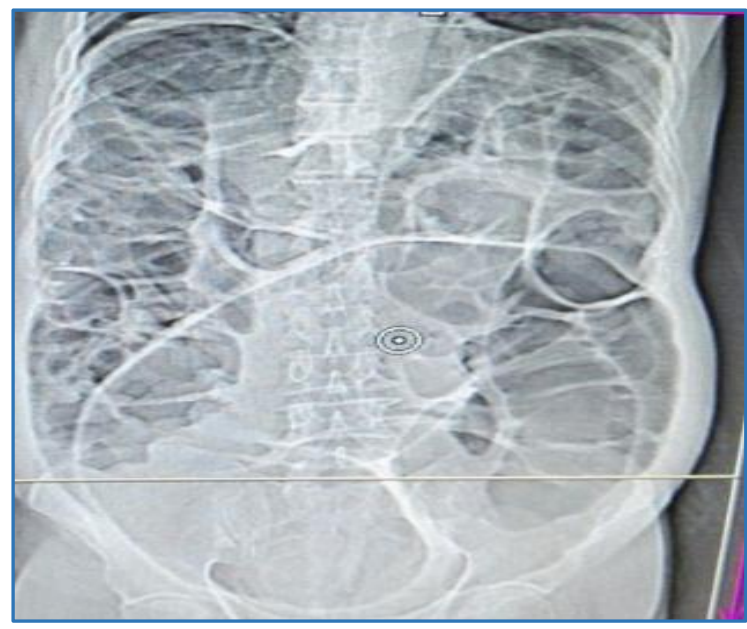

Image 2: CECT Abdomen and Pelvis showing Sigmoid Volvulus 


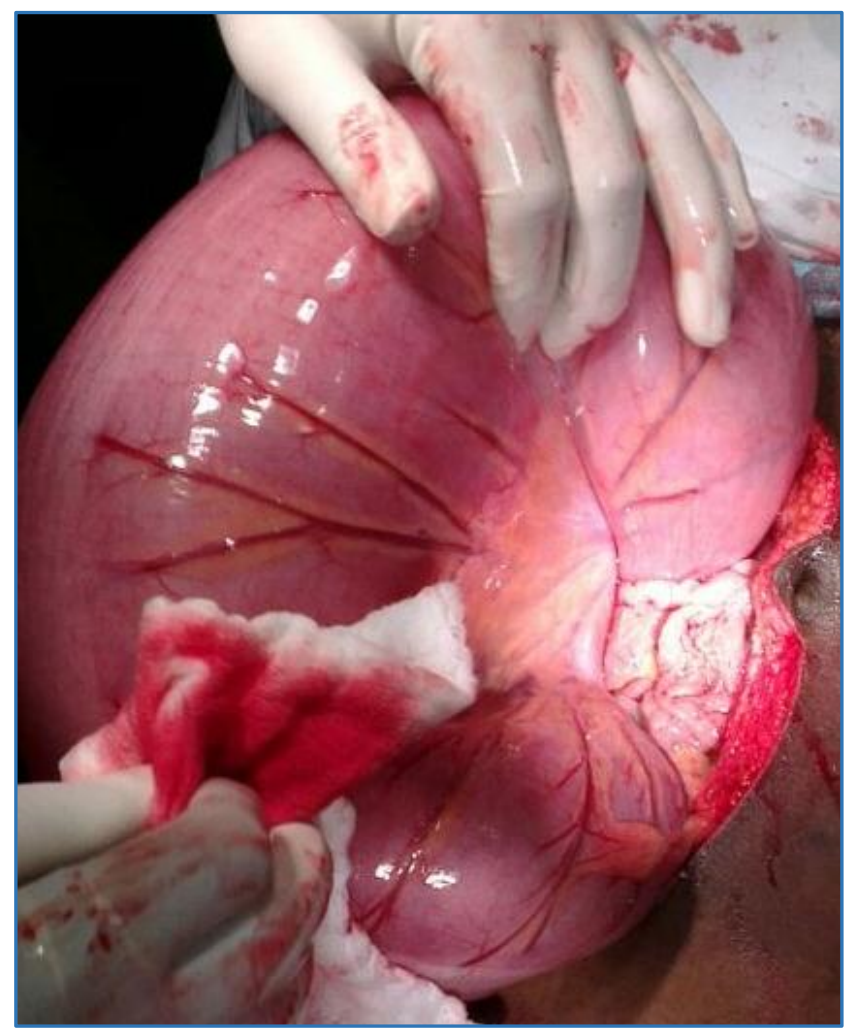

Image 3: Intra-op finding of Sigmoid Volvulus

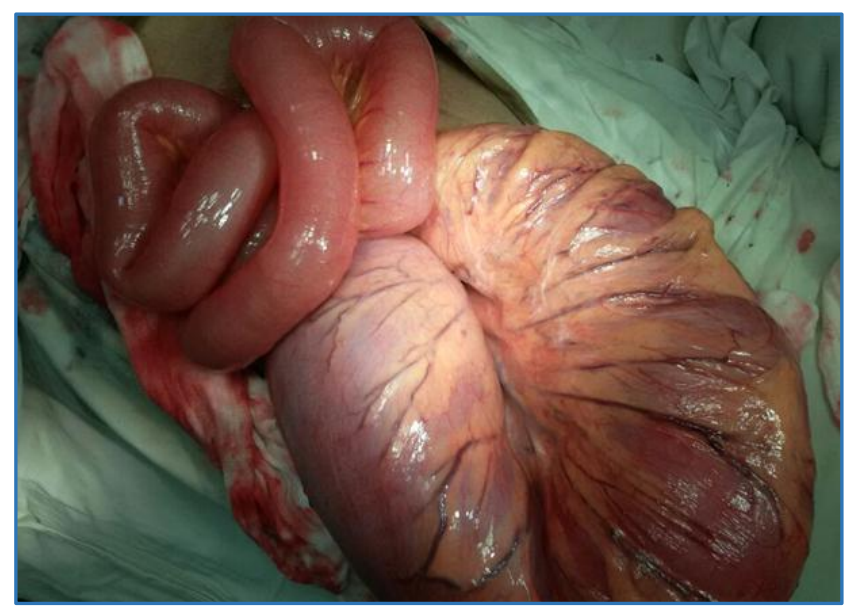

Image 4: Intra-op Finding of Sigmoid Volvulus showing Dilated Sigmoid and Proximal Bowel Loops

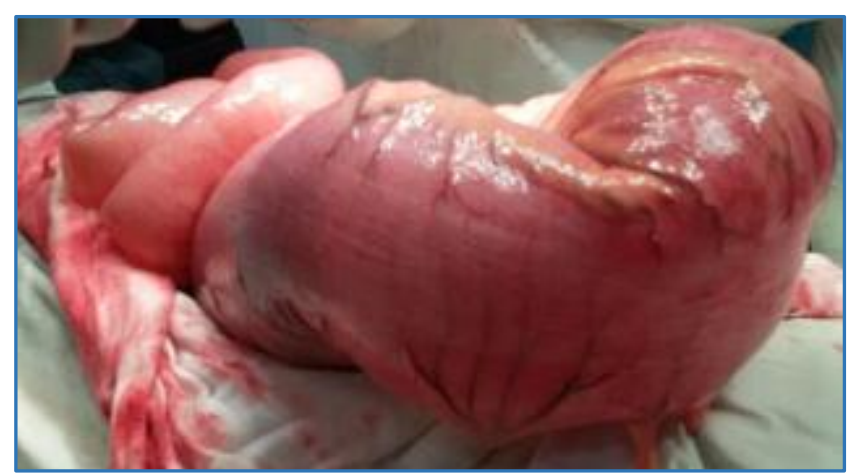

Image 5: Intra-op finding of Sigmoid Volvulus

\section{DISCUSSION}

Volvulus is defined as a condition in which a segment of the bowel becomes twisted on its mesenteric axis causing partial or complete obstruction (Image 3,4 and 5). Compromise in the blood supply together with increase in the intraluminal pressure leads to perforation or gangrene if left untreated.12 Volvulus is more common in males than in females. Women are thought to have lower incidence due to wider pelvis. ${ }^{9}$ In our study, male:female ratio is $2.5: 1$.

There are various potential sites where a volvulus can form. These include: Sigmoid volvulus (80\% cases), caecal volvulus (15\%), transverse colon volvulus (3\%) and splenic flexure volvulus $(2 \%){ }^{13}$

The details of sigmoid volvulus were found in the ancient Roman and Greek writings and in the Egyptian papyrus. Untwisting of the sigmoid volvulus by insufflations with air as a treatment which was advocated by Hippocrates is still being used as a nonoperative mode of treatment of sigmoid volvulus.

There are several causes of sigmoid volvulus, most of which are acquired. The volvulus is likely to develop as a consequence of mesocolon lengthening by gradual elongation of sigmoid intestine. This leads to redundant and long sigmoid colon predisposing to sigmoid volvulus.9,13 Decrease in the enteric plexuses and ganglion cells are also associated with sigmoid volvulus. ${ }^{4}$

The aim of management includes resuscitation, reduction of volvulus, relief of obstruction and prevention of recurrence. Third space losing, hypovolaemia and shock may require stabilising patient with fluid resuscitation. Diagnosis is usually based on physical examination and radiographic investigations.

Clinical symptoms include abdominal distension, pain abdomen, vomiting and constipation. In our study, abdominal distension was the most common symptom [100\%], followed by constipation [91.42\%].

Air fluid levels in each segments of the dilated bowel, coffee bean sign/bent inner tube sign (Image 1 and 2) with central cleft of bean in sigmoid volvulus and absence of gas in rectum are the radiological signs of sigmoid volvulus.13,14,15,16 Treatment includes detorsion by sigmoidoscopy or transrectal intubation as described by Brusgaard carries risk of recurrence. ${ }^{17}$

If detorsion fails or in case of clinical evidence or suspicion of perforation, emergency laparotomy is essential. Various procedures have been adopted at laparotomy in the emergency management of sigmoid volvulus. Permanent cure of the sigmoid volvulus is by resection of sigmoid colon and anastomosis. Single stage resection and anastomosis ensures permanent cure, reduces morbidity and mortality, and shortens duration of hospital stay is also desirable. ${ }^{18,19}$

In our study too, primary resection with anastomosis was found to be successful [100\%] as compared to sigmoidopexy or Hartmann's procedure. The overall mortality rate was found to be $11.42 \%$.

\section{CONCLUSION}

Sigmoid volvulus is a rare but an important cause of large bowel obstruction, a surgical emergency requiring prompt diagnosis and treatment by detorsion before patient develops complications such as gangrene or perforation. Primary resection and anastomosis is a permanent curative procedure for sigmoid volvulus. 


\section{REFERENCES}

1. Bolt DE. The management of volvulus of sigmoid colon. $\mathrm{Br}$ J Surg 1956;44(184):172-5.

2. Raveenthiran R, Madiba TE, Atamanalp SS, et al. Volvulus of the sigmoid colon. Colorectal Dis 2010;12(7 Online):e $1-17$.

3. Atamanalp SS, Yildirgan MI, Basoglu M, et al. Sigmoid colon volvulus in children: review of 19 cases. Pediatr Surg Int 2004;20(7):492-5.

4. Hinshaw DB, Carter R. Surgical management of acute volvulus of the sigmoid colon: a study of 55 cases. Ann Surg 1957;146(1):52-60.

5. Lal SK, Morgenstern R, Vinjrayer EP, et al. Sigmoid volvulus: an update. Gastrointest Endoscopy Clin N Am 2006;16(1):175-87.

6. Arnold GJ, Nance FC. Volvulus of the sigmoid colon. Ann Surg 1973;177(5):527-37.

7. String ST, DeCosse JJ. Sigmoid volvulus. An examination of the mortality. Am J Surg 1971;121(3):293-7.

8. Sriram K, Schumer W, Ehrenpresis S, et al. Phenothiazine effect on gastrointestinal tract function. Am J Surg 1979;137(1):87-91.

9. Mangiante EC, Croce MA, Fabian TC, et al. Sigmoid volvulus. A four-decade experience. Am Surg 1989;55(1):41-4.

10. Anderson JR, Lee D. The management of acute sigmoid volvulus. Br J Surg 1981;68(2):117-20.
11. Ballantyne GH, Brandner MD, Beart RW, et al. Volvulus of the colon. Incidence and mortality. Ann Surg 1985;202(1):83-92.

12. Machado NO. Lleosigmoid knot: a case report and literature review of 280 cases. Ann Saudi Med 2009;29(5):402-6.

13. Theuer C, Cheadle WG. Volvulus of the colon. Am Surg 1991;57(3):145-50.

14. Fujiya K, Chong JM, Ando M, et al. Sigmoid volvulus is associated with a decrease in enteric plexus and ganglion cells: a case-control study. Int J Colorectal Dis 2015;30(5):673-8.

15. Salati U, McNeill G, Torregiani WC. The coffee bean sign in sigmoid volvulus. Radiology 2011;258(2):651-2.

16. William M, Steffes CP. Sigmoid volvulus in a 46 year old man. Hospital Physician 2006;42(1):33-36.

17. Madiba TE, Thomson SR. The management of sigmoid volvulus. J Roy Coll Surg Edinb 2000;45(2):74-80.

18. Sule AZ, Lya D, Obekpa PO, et al. One-stage procedure in the management of acute sigmoid volvulus. J Roy Coll Edinb 1999;44(3):164-6.

19. Bhatnager BN, Sharma CL. Nonresective alternative for the cure of non-gangrenous sigmoid volvulus. Dis Colon Rectum 1998;41(3):381-8. 